\title{
A compact dusty disk around the Herbig Ae star HR 5999 resolved with VLTI / MIDI ${ }^{\star}$
}

\author{
Th. Preibisch ${ }^{1}$, S. Kraus ${ }^{1}$, Th. Driebe ${ }^{1}$, R. van Boekel $^{2}$, and G. Weigelt ${ }^{1}$ \\ 1 Max-Planck-Institut für Radioastronomie, Auf dem Hügel 69, 53121 Bonn, Germany \\ e-mail: preib@mpifr-bonn.mpg.de \\ 2 Max-Planck-Institut für Astronomie, Königstuhl 17, 69117 Heidelberg, Germany
}

Received 3 February 2006 / Accepted 22 June 2006

ABSTRACT

\begin{abstract}
Aims. We have used mid-infrared long-baseline interferometry to resolve the circumstellar material around the Herbig Ae star HR 5999, providing the first direct measurement of its angular size, and to derive constraints on the spatial distribution of the dust. Methods. MIDI at the VLTI was used to obtain a set of ten spectrally dispersed $(8-13 \mu \mathrm{m})$ interferometric measurements of HR 5999 at different projected baseline lengths and position angles. To derive constraints on the geometrical distribution of the dust, we compared our interferometric measurements to $2 \mathrm{D}$, frequency-dependent radiation transfer simulations of circumstellar disks and envelopes.

Results. The derived visibility values between $\sim 0.5$ and $\sim 0.9$ show that the mid-infrared emission from HR 5999 is clearly resolved. The characteristic size of the emission region depends on the projected baseline length and position angle, and it ranges between 5-15 milliarcsec (Gauss $F W H M$ ), corresponding to remarkably small physical sizes of $\sim 1-3$ AU. For disk models with radial power-law density distributions, the relatively weak but very extended emission from outer disk regions ( $\gtrsim 3$ AU) leads to model visibilities that are significantly lower than the observed visibilities, making these models inconsistent with the MIDI data. Disk models in which the density is truncated at outer radii of $\sim 2-3 \mathrm{AU}$, on the other hand, provide good agreement with the data.

Conclusions. A satisfactory fit to the observed MIDI visibilities of HR 5999 is found with a model of a geometrically thin disk that is truncated at $2.6 \mathrm{AU}$ and seen under an inclination angle of $58^{\circ}$ (i.e. closer to an edge-on view than to a face-on view). Neither models of a geometrically thin disk seen nearly edge-on, nor models of spherical dust shells can achieve agreement between the observed and predicted visibilities. The reason why the disk is so compact remains unclear; we speculate that it has been truncated by a close binary companion.
\end{abstract}

Key words. techniques: interferometric - stars: individual: HR 5999 - stars: formation - stars: circumstellar matter

\section{Introduction}

Herbig AeBe stars (HAEBEs; see Herbig 1960, 1994; Thé et al. 1994) are pre-main sequence stars of intermediate mass $\left(\sim 2-10 M_{\odot}\right)$ that show broad emission lines, rapid variability, and excess infrared and millimeter-wavelength emission. These properties suggest the presence of substantial amounts of circumstellar dust and gas. The distribution of the circumstellar material is still a matter of debate. Since until recently the spatial scales of the inner circumstellar environment (a few AU, corresponding to $\lesssim 0.1^{\prime \prime}$ ) were not accessible to optical and infrared imaging observations, conclusions drawn on the spatial distribution of the circumstellar material of the HAEBE stars were in most cases based entirely on the modeling of the spectral energy distribution (SED). However, fits to the observed SEDs are highly ambiguous (e.g. Men'shchikov \& Henning 1994). For example, the SEDs of some HAEBEs could be equally well explained with such very different models as geometrically thin accretion disks and spherical envelopes (Hillenbrand et al. 1992; Miroshnichenko et al. 1997). More complex models, like envelopes with cavities produced by bipolar molecular outflows (Hartmann et al. 1993), geometrically thin and optically thick

* Based on observations with the ESO Very Large Telescope Interferometer at Paranal Observatory under programme

IDs 073C-0248 and 073C-0720. disks surrounded by a spherical envelope (Natta \& Krügel 1995; Natta et al. 2001; Miroshnichenko et al. 1999), flared outer disks, puffed-up inner disk rims (Dullemond et al. 2001), and disk plus inner halo models (Vinkovic et al. 2006) have also been used to successfully fit the observed SEDs of HAEBEs. The strongest evidence for circumstellar disks around HAEBEs comes from millimeter interferometry (Mannings \& Sargent 1997, 2000): flattened structures around several sources have been resolved on $\sim 100 \mathrm{AU}$ scales at millimeter wavelengths and detailed kinematic modeling of one source, MWC 480, is consistent with rotation in a Keplerian disk.

Speckle interferometry by Leinert et al. (2001) in the NIR reveals numerous examples of resolved halos on scales of $\sim 0.1^{\prime \prime}-1^{\prime \prime}$ (corresponding to several hundred AU). Bispectrum speckle interferometry revealed complex asymmetric structures on scales down to 70 mas in several intermediate- to highmass young stellar objects (e.g. Schertl et al. 2000; Weigelt et al. 2002a,b, 2006; Preibisch et al. 2001, 2002, 2003; Hofmann et al. 2004). Interferometric aperture masking Keck observations of the young system $\mathrm{LkH} \alpha 101$ (Tuthill et al. 2001, 2002) revealed some details of the inner disk, which is seen nearly faceon. To make further progress on this topic, observations with even higher spatial resolution, at the level $\lesssim 10$ milliarcsec (mas), are needed to explore the inner circumstellar regions of these objects in detail. Such observations can only be performed with long-baseline interferometry. 
The current generation of infrared long-baseline interferometers is sensitive to the distribution of dust around the nearest young stars on scales on the order of $1 \mathrm{AU}$, and it provides a powerful probe of the models of disks and envelopes of such stars. The first systematic study of HAEBEs using the technique of long-baseline interferometry in the near-infrared was conducted by Millan-Gabet et al. (2001), who could resolve the near-infrared emission of 11 of the 15 HAEBEs observed at the IOTA interferometer. The derived sizes of the observed structures are 0.5 to $6 \mathrm{AU}$, but no unique geometrical model could be determined for any of the sources due to the very sparse coverage of the $(u, v)$-plane. A number of further studies have been obtained at different interferometers (e.g., Akeson et al. 2000; Wilkin \& Akeson 2003; Eisner et al. 2004; Monnier et al. 2005). Most of the near-infrared interferometric data are consistent with a simple disk model possessing a central dust-free cavity, ringed by hot dust, but other geometries can often not be excluded, due to the poor sampling of these observations of the $(u, v)$-plane.

The first long-baseline interferometric observations of HAEBEs at mid-infrared wavelengths were recently presented by Leinert et al. (2004). Their data, obtained with MIDI at the VLTI, trace the $8-13 \mu \mathrm{m}$ emission from hot and warm dust in the inner disk regions and can be used to constrain the geometrical structure of the circumstellar material on angular scales of $\sim 10$ mas. They resolved the circumstellar material around all of the seven observed, nearby HAEBEs and derived characteristic dimensions of the emitting regions at $10 \mu \mathrm{m}$ ranging from $1 \mathrm{AU}$ to $10 \mathrm{AU}$, but, again, they could not determine the geometry of the dust distributions due to the limited $(u, v)$-coverage of the observations.

HR 5999 (=HD $144668=$ V856 Sco) is one of the best studied HAEBEs. It lies in the central part of the Lupus 3 dark cloud, which is part of the extended Lupus star-formation complex. HR 5999 has a spectral type of A7 III-IV and forms a $\sim 45^{\prime \prime}$ wide proper-motion binary system with the A1.5 star HR 6000 . The Hipparcos parallax for HR 5999 corresponds to a distance of $208_{-30}^{+50} \mathrm{pc}$. The spectral energy distribution of HR 5999 shows a clear infrared excess at wavelengths above $1 \mu \mathrm{m}$. IRAS detected HR 5999 at 12,25 , and $60 \mu \mathrm{m}$, but provided only an upper limit at $100 \mu \mathrm{m}$. Henning et al. (1994) found weak millimeter emission from HR 5999, but the detection was only at the $1 \sigma$ significance level. The far-infrared and mm-emission of HR 5999 suggests the total mass of the circumstellar material to be on the order $0.006 M_{\odot}$ (Siebenmorgen et al. 2000). HR 5999 belongs to the "group I objects" as defined by Hillenbrand (1992); the infrared excess of these objects is thought to be from emission arising in a flat, optically thick accretion disk. The fit of the observed SED with a simple analytical model of an optically thick circumstellar disk yielded an outer radius of $\gtrsim 23 \mathrm{AU}$ and a possible inner hole of $\sim 0.1 \mathrm{AU}$. Siebenmorgen et al. (2000) analyzed mid-infrared ISO spectra of HR 5999 and found a broad silicate emission feature with a peak around $9.6 \mu \mathrm{m}$. With a ratio of peak- to continuum-flux of about 1.4 , the intensity of this feature is relatively small. The broad and rather flat shape of the silicate feature indicates the presence of relatively large $(\gtrsim 1 \mu \mathrm{m})$ silicate grains (cf. van Boekel et al. 2003).

The stellar parameters of HR 5999 suggest that the object is $\sim 0.5$ Myr old and has a mass of $\sim 3-4 M_{\odot}$ (van Boekel et al. 2005). HR 5999 will thus arrive at the main sequence as a mid B-type star. HR 5999 shows only moderate extinction $\left(A_{\mathrm{V}}=0.49 \mathrm{mag}\right)$ and is a fast rotator $\left(v \sin i=204 \mathrm{~km} \mathrm{~s}^{-1}\right.$; Royer et al. 2002). The quasi-periodic and irregular photometric and spectroscopic variability of HR 5999 in the optical (see Perez et al. 1992) and in the UV range (see Perez et al. 1993) has been
Table 1. Log of MIDI observations of HR 5999.

\begin{tabular}{llrcc}
\hline \hline UT date and time & $\begin{array}{r}B \\
{[\mathrm{~m}]}\end{array}$ & $\begin{array}{r}\text { PA } \\
{[\mathrm{deg}]}\end{array}$ & $\begin{array}{c}\text { Obs. } \\
\text { name }\end{array}$ \\
\hline $2004-04-10$ & $02: 44: 34$ & 46.34 & 173.37 & $46 \mathrm{~m} / 173^{\circ}$ \\
$2004-04-10$ & $05: 23: 56$ & 46.60 & 21.57 & $46 \mathrm{~m} / 21^{\circ}$ \\
$2004-04-11$ & $05: 16: 58$ & 46.59 & 20.74 & $46 \mathrm{~m} / 20^{\circ}$ \\
$2004-04-11$ & $09: 09: 04$ & 42.42 & 52.12 & $42 \mathrm{~m} / 52^{\circ}$ \\
$2004-04-12$ & $09: 46: 20$ & 39.76 & 56.36 & $39 \mathrm{~m} / 56^{\circ}$ \\
$2004-06-28$ & $00: 15: 43$ & 102.38 & 15.35 & $102 \mathrm{~m} / 15^{\circ}$ \\
$2004-06-28$ & $02: 06: 06$ & 100.76 & 30.90 & $100 \mathrm{~m} / 30^{\circ}$ \\
$2004-06-28$ & $04: 26: 08$ & 90.89 & 46.10 & $90 \mathrm{~m} / 46^{\circ}$ \\
$2004-06-28$ & $05: 22: 28$ & 83.22 & 50.57 & $83 \mathrm{~m} / 50^{\circ}$ \\
$2004-09-30$ & $00: 02: 18$ & 63.50 & 123.74 & $63 \mathrm{~m} / 123^{\circ}$ \\
\hline
\end{tabular}

interpreted as due to instabilities in an optically thick circumstellar accretion disk. The detailed analysis of the UV emission lines in the IUE spectra of HR 5999 by Perez et al. (1993) revealed gas accreting onto the star with velocities as high as $300 \mathrm{~km} \mathrm{~s}^{-1}$ (see also Blondel et al. 1993). Perez et al. (1993) also concluded that the observed double-peaked $\mathrm{H} \alpha$ and $\mathrm{Mg}$ II emission profiles strongly suggest that the circumstellar disk around HR 5999 is seen nearly edge-on. The average mass accretion rate was estimated to be $\sim 7 \times 10^{-7} M_{\odot} / y r$, but the observed variability of the UV emission clearly suggests that the accretion process happens in a non-steady fashion.

To summarize, the existing data clearly show that HR 5999 is surrounded by circumstellar material, probably in the form of an accretion disk. The high $v \sin i$ and the double-peaked structure of the UV emission lines suggest that the system is seen under a relatively high inclination angle (i.e. close to edge on). This makes it an interesting target for a detailed interferometric study of the geometry of the circumstellar dust. Grady et al. (2005) presented HST STIS white-light coronographic imaging results for HR 5999, found no indication of nebulosity at $r>70 \mathrm{AU}$, and concluded that the disk around HR 5999 must be geometrically small.

HR 5999 has a close visual companion, Rossiter 3930 (see Stecklum et al. 1995, and references therein), with $\Delta V \sim 4.6$ and $\Delta K \sim 3.6$, at a projected separation of 1.4", corresponding to a projected distance of about 300 AU. Since Rossiter 3930 shows strong $\mathrm{H} \alpha$ emission, it is very likely a T Tauri star. If Rossiter 3930 is actually physically related to HR 5999 and not a chance projection, it may be responsible for the rather low (sub-)mm emission and corresponding low mass of the circumstellar material of HR 5999 due to the mechanism of dynamic disk clearing by gravitational binary interaction (e.g. Arymowicz \& Lubow 1994).

\section{Observations and data analysis}

The observations discussed in this paper were obtained with the MIDI instrument at the VLTI during several observing runs between April and September 2004. In total, ten MIDI measurements of HR 5999 were performed: six visibility measurements for observing program 073C-0248 (PI: Preibisch) and four visibility measurements for program 073C-0720 (PI: van Boekel). The combined data set is analyzed in this paper. The observation dates, the projected baseline lengths, the position angles of the projected baseline on the sky, and the abbreviated data set names used in the following text are summarized in Table 1. Our observations cover a range of projected baseline lengths from $39 \mathrm{~m}$ to 
Table 2. List of calibrators used for the calibration of the HR 5999 data together with uniform-disk diameters $\left(d_{\mathrm{UD}}\right)$ and the date, as well as the time stamp $\left(t_{\mathrm{obs}}\right)$ of MIDI observations.

\begin{tabular}{rccc}
\hline \hline $\begin{array}{r}\text { Calibrator } \\
\text { HD number }\end{array}$ & $d_{\text {UD }}($ mas) & $\begin{array}{c}\text { Night } \\
(2004)\end{array}$ & $t_{\text {obs }}($ UTC $)$ \\
\hline 81797 & $9.54 \pm 0.75$ & $2004-04-10$ & $00: 41: 19$ \\
& & $2004-04-11$ & $03: 56: 19$ \\
\hline 107446 & $4.54 \pm 0.23$ & $2004-04-10$ & $02: 13: 31$ \\
& & $2004-04-11$ & $23: 55: 03$ \\
& & $2004-04-12$ & $01: 24: 27$ \\
\hline 109379 & $3.30 \pm 0.17$ & $2004-06-27$ & $23: 27: 48$ \\
\hline 129456 & $3.39 \pm 0.09$ & $2004-04-10$ & $05: 46: 05$ \\
& & $2004-04-12$ & $08: 37: 42$ \\
& & $2004-04-12$ & $10: 09: 08$ \\
& & $2004-06-28$ & $00: 43: 13$ \\
\hline 134505 & $2.55 \pm 0.13$ & $2004-06-28$ & $01: 43: 12$ \\
\hline 139127 & $3.30 \pm 0.22$ & $2004-04-11$ & $05: 35: 48$ \\
& & $2004-04-11$ & $09: 36: 42$ \\
& & $2004-04-12$ & $07: 09: 25$ \\
& & $2004-06-28$ & $02: 48: 50$ \\
& & $2004-06-28$ & $05: 43: 29$ \\
\hline 152885 & $2.88 \pm 0.09$ & $2004-04-10$ & $07: 13: 48$ \\
& & $2004-04-12$ & $05: 57: 26$ \\
& & $2004-04-12$ & $09: 24: 41$ \\
\hline 161892 & $3.94 \pm 0.21$ & $2004-04-12$ & $04: 42: 03$ \\
& & $2004-04-12$ & $05: 02: 42$ \\
\hline 168723 & $2.88 \pm 0.13$ & $2004-04-10$ & $09: 29: 56$ \\
& & $2004-04-10$ & $10: 12: 13$ \\
& & $2004-09-30$ & $01: 22: 01$ \\
\hline 169916 & $3.39 \pm 0.09$ & $2004-04-12$ & $07: 57: 12$ \\
\hline 176411 & $2.24 \pm 0.13$ & $2004-04-10$ & $08: 08: 34$ \\
& & $2004-04-10$ & $08: 45: 41$ \\
\hline 188512 & $2.07 \pm 0.10$ & $2004-09-30$ & $03: 13: 45$ \\
\hline & & &
\end{tabular}

$102 \mathrm{~m}$ and position angles ranging from $15^{\circ}$ to $173^{\circ}$, and, therefore, provide a relatively good coverage of the $(u, v)$ plane.

The fringes were scanned by applying rapid internal optical path difference (OPD) changes of a few wavelengths around the point of zero OPD. A prism with a spectral resolution of $\lambda / \Delta \lambda \simeq 30$ was used to obtain spectrally dispersed fringes in the range of 8 to $13 \mu \mathrm{m}$. Immediately after a recording of interferometric data, photometric calibration data were obtained by blocking each of the two beams alternately. Chopping with a frequency of $2 \mathrm{~Hz}$ was applied to obtain the data for the sky background subtraction. A more detailed description of the observing procedure is given in Przygodda et al. (2003), Leinert et al. (2004), Chesneau et al. (2005a,b), and Ohnaka et al. (2005).

Calibration stars with known angular diameter were observed for the calibration of the raw visibilities. Table 2 gives an overview of these calibration stars, their uniform-disk diameters as given in the CalVin list ${ }^{1}$, and the MIDI observations that we used for the calibration of the raw visibilities of HR 5999.

We utilized two different MIDI data reduction packages: MIA developed at the Max-Planck-Institute for Astronomy and EWS developed at the Leiden Observatory. While the MIA package is based on the power spectrum analysis and measures the total power of the observed fringes, the EWS package first corrects for OPDs (instrumental, as well as atmospheric delays) in each scan, and then the interferograms are averaged. A more detailed description of the MIA and EWS packages can be found in Leinert et al. (2004) and Jaffe (2004), respectively. In our analysis of the HR 5999 data, we found good agreement between the results of both packages within the error bars. In the following

\footnotetext{
${ }^{1}$ Available from ESO at http://www. eso.org/observing/etc/
}

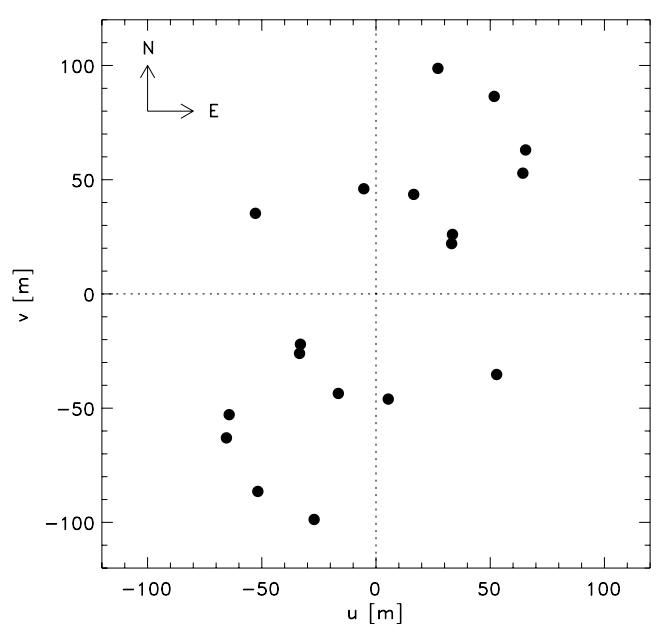

Fig. 1. Points in the $(u, v)$ plane sampled by our MIDI observations of HR 5999.

discussion we always refer only to the results obtained with the MIA software.

The errors of the calibrated visibility were estimated from the $1 \sigma$ scatter of the visibilities calibrated with all individual data sets of a night. In addition, to account for systematic error contributions (e.g. due to imperfect beam overlap), we assumed a minimum visibility error of $15 \%$. One of the ten visibility measurements, the $46 \mathrm{~m} / 21^{\circ}$ data set, was affected by strong variations in the transfer function around the time of the measurement. This particular data set could not be calibrated well; we decided to drop it from the following analysis.

\section{Observed visibilities}

As the first step of the analysis, we consider the dependence of the observed visibilities on the wavelength, the projected baseline length, and the baseline position angle. Figure 2 shows the wavelength-dependence of the visibilities of HR 5999. All visibility curves are rather flat, i.e. the observed visibilities show no strong dependence on the wavelength.

Figure 3 shows the observed $10.5 \mu \mathrm{m}$ visibilities as a function of projected baseline length. One can see that the data are not consistent with simple uniform-disk or Gaussian brightness distribution models.

\subsection{Size of the emission region}

In order to derive an initial rough estimate of the size of the resolved emission region, we fitted each observed visibility value with two simple models: first, a uniform-disk model, and second, a Gaussian brightness distribution. The resulting diameters range between 10 and 25 milliarcsec (corresponding to $\sim 2-5 \mathrm{AU}$ at the distance of $208 \mathrm{AU}$ ) for the uniform disk model and 5-15 mas ( 1-3 AU) for the Gauss model (FWHM). The Gauss-fit diameters as a function of wavelength for the different MIDI observations are shown in Fig. 4. For all data sets, the diameters increase with increasing wavelength in the MIDI band. This effect can be understood as a consequence of the fact that the emission at longer wavelengths comes from cooler material, which is located at larger distances from the central star than the warmer material radiating at shorter wavelengths. Typically, the diameters at $13 \mu \mathrm{m}$ are $\sim 50 \%-100 \%$ larger than at $8 \mu \mathrm{m}$. 

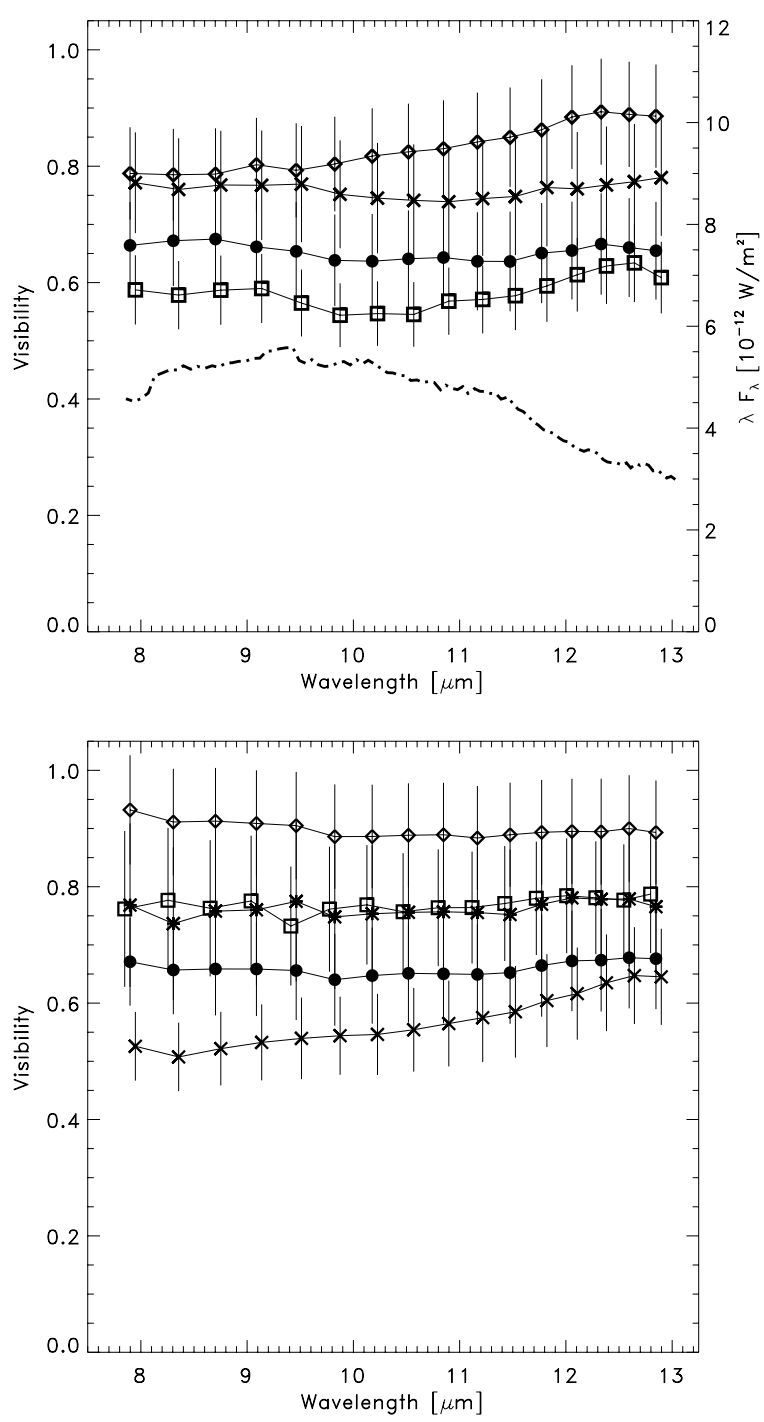

Fig. 2. MIDI visibilities of HR 5999 plotted versus wavelength. The upper panel shows the data for the observations at baselinelength/position angles of $102 \mathrm{~m} / 15^{\circ}$ (dots), $83 \mathrm{~m} / 50^{\circ}$ (crosses), $63 \mathrm{~m} / 123^{\circ}$ (boxes), and $46 \mathrm{~m} / 20^{\circ}$ (diamonds). The lower panel shows the data for the observations at $100 \mathrm{~m} / 30^{\circ}$ (dots), $90 \mathrm{~m} / 46^{\circ}$ (crosses), $46 \mathrm{~m} / 173^{\circ}$ (boxes), $42 \mathrm{~m} / 52^{\circ}$ (diamonds), and $39 \mathrm{~m} / 56^{\circ}$ (asterisks). The thick dash-dotted line and the corresponding scale on the right $y$-axis in the upper panel show the flux-calibrated spectrum obtained with MIDI during the $39 \mathrm{~m} / 56^{\circ}$ observation.

Another notable effect is the trend that the longest baselines give the smallest diameters. Although one must not ignore that the different observations were obtained at different position angles (see next paragraph), this suggests a complex geometry for the emission region, as discussed in the next section.

\subsection{Shape of the emission region}

In Fig. 5 we plot the Gauss-fit radii (at wavelength $10.5 \mu \mathrm{m}$ ) as a function of the position angle of the observation. The plot suggests that the structure of the emission region is not described well by a spherically symmetric distribution, i.e. seems to be asymmetric. An inclined ellipse with axis ratio 1:1.56 apparently yields better agreement to the data than a circle, but does not provide a particularly good fit either. The data suggest that the emission has a more complicated structure, probably with

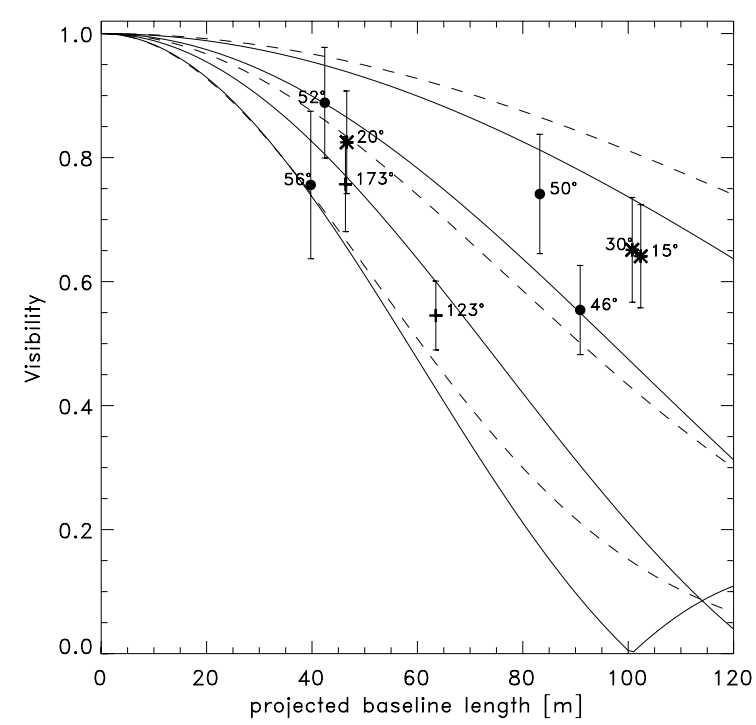

Fig. 3. Visibility at $10.5 \mu \mathrm{m}$ as a function of projected baseline length. The data points are annotated with their respective position angles. For comparison, we also plotted the theoretical visibility curves for uniform disk models with diameters of 10, 15, 20, and 25 mas (solid lines) and for Gauss models with $F W H M=5,10$, and 15 mas (dashed lines).

substructure at different size scales. A more detailed analysis of size and shape of the emission region is described below in Sect. 4.

\subsection{Comparison to MIDI data of other HAEBES}

The visibilities measured for HR 5999 differ from those obtained by Leinert et al. (2004) for a sample of seven HAEBEs in two ways. First, all MIDI measurements of HR 5999 give visibilities $\geq 0.5$, whereas six of the seven HAEBEs in the Leinert et al. (2004) sample show much lower visibility values, $\leq 0.4$ in the $8 \mu \mathrm{m}$ to $13 \mu \mathrm{m}$ wavelength range. Only 51 Oph shows visibility values similar to what we find for HR 5999. This implies that the angular extent of the mid-infrared emission from HR 5999 is smaller than that of most HAEBEs in the Leinert sample.

The second difference is the wavelength dependence of the visibilities. While the visibility for HR 5999 is essentially flat over the $8 \mu \mathrm{m}$ to $13 \mu \mathrm{m}$ wavelength range, most of the HAEBE stars in the Leinert sample show a rather strong drop in the visibility between $8 \mu \mathrm{m}$ and $10 \mu \mathrm{m}$, in some cases followed by a slight increase towards longer wavelengths. The latter wavelength dependence of the visibility is expected for objects with significant $10 \mu \mathrm{m}$ silicate emission features (a strong emission feature in the spectrum produces a minimum in the visibility due to optical depth effects, because the apparently larger object size in the center of the emission line yields lower visibilities). The flat visibility curves of HR 5999 are probably related to its very weak silicate emission feature, which does not contribute enough to produce a significant dependence of the visibility (apparent object size) on wavelength over this feature.

\section{Constraints on the source shape from 2D radiative transfer simulations}

The true morphology of the mid-infrared emission is most likely more complex than simple geometrical models for the brightness distribution, like uniform-disk or Gauss models. For example, the existence of a dust-free hole inwards of the dust 


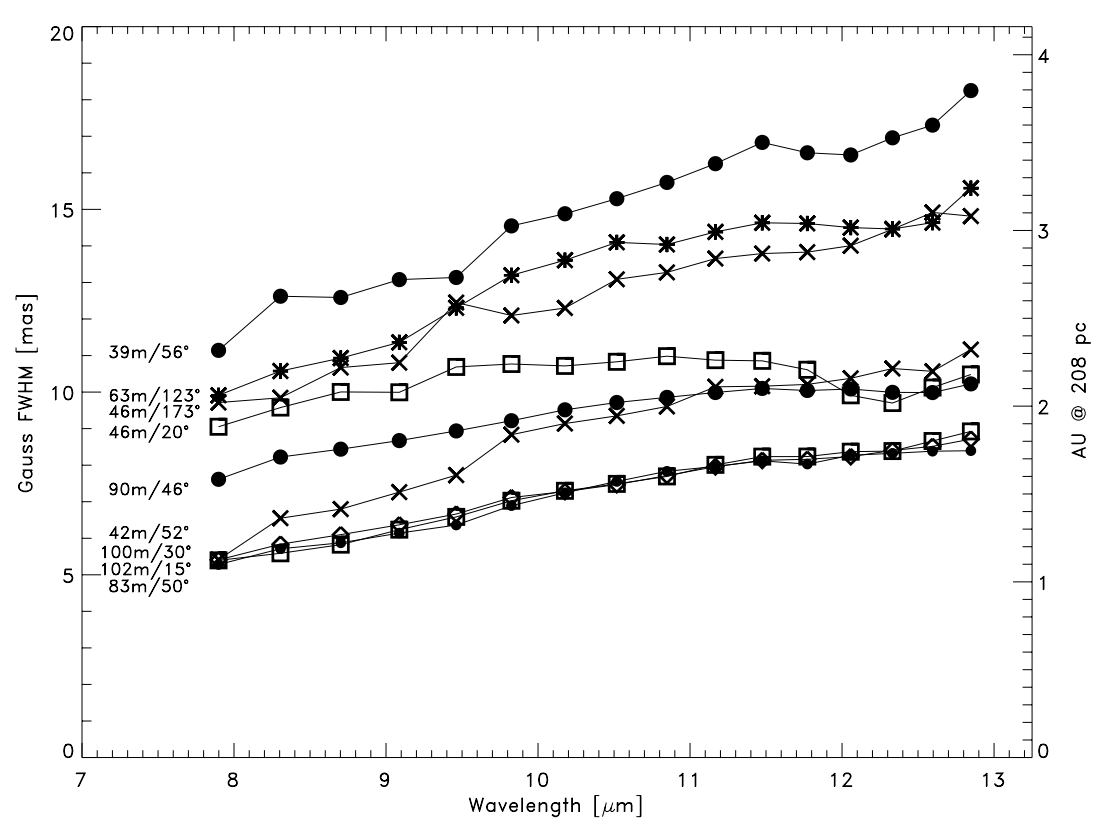

Fig. 4. Gauss-fit $F W H M$ diameters as a function of wavelength derived for the MIDI observations of HR 5999. The individual observations are identified by their values for projected baseline length and position angle.

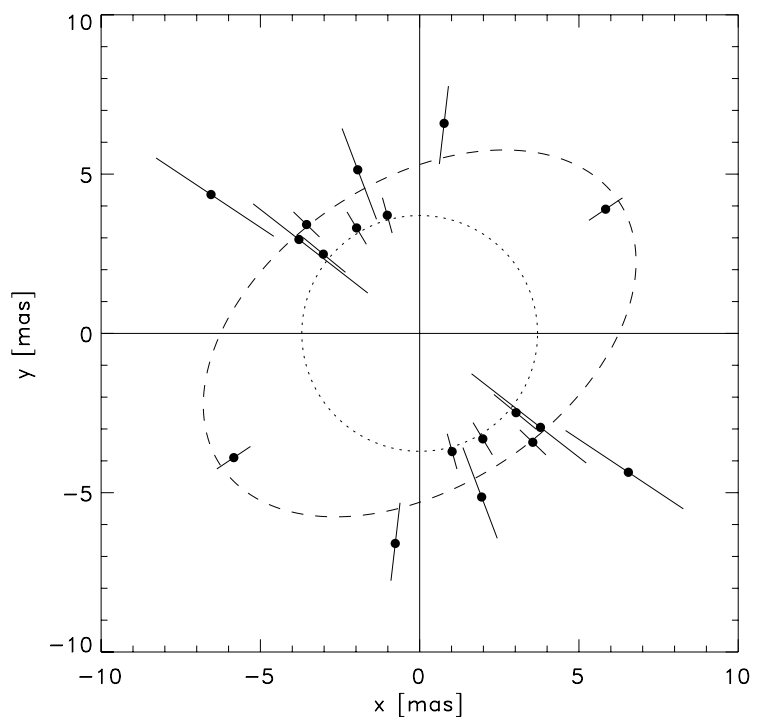

Fig. 5. Gauss-fit $F W H M$ diameters at $10.5 \mu \mathrm{m}$ for the different observations as a function of baseline position angle. The dotted circle has a radius of 3.7 mas. The dashed ellipse has a minor axis of 4.8 mas and a major axis of 7.5 mas.

sublimation radius already implies that the brightness distribution cannot have a steadily decreasing profile. In order to see how much information on the spatial distribution of circumstellar material can be deduced from our MIDI data, we performed $2 \mathrm{D}$ radiation transfer calculations. We believe that this approach provides more realistic constraints than the use of simple analytical models. Our radiative transfer modeling also includes a self-consistent determination of the dust sublimation radius, i.e. the inner disk edge, which is a crucial ingredient in any realistic modeling attempt. Note that the aim of the modeling in this paper is an exploration of different geometries for the dust distribution and not a detailed disk modeling (which is not warranted considering the rather large uncertainties of the measured visibilities).

\subsection{Modeling procedure}

For our simulations we used the $2 \mathrm{D}$ radiation transfer code described in detail by Sonnhalter et al. (1995). In this code, the distribution of dust temperatures and radiation intensities in an axially symmetric dusty circumstellar environment around a central radiation source is calculated within the framework of the flux-limited diffusion approximation (Levermore \& Pomraning 1981). The equations are discretized on a quadratic, equally spaced grid. As the density distribution is assumed to be axially symmetric with respect to the $z$-axis and mirror symmetric with respect to the plane $z=0$, cylindrical coordinates $(r, z, \phi)$ are used, because the symmetry reduces the relevant information to the first quadrant of the $r-z$ plane. To improve resolution and convergence, a system of 5 nested grids with decreasing grid spacing is used. The differential equations for the radiation field are iterated together with the temperature equations until a self-consistent equilibrium configuration is reached. At the end of the iteration procedure, the total radiation flux (integrated over all frequencies) through the outermost grid cells was always within $1 \%$ of the stellar luminosity for all computed models. We note that any non-spherical density distribution results in non-spherically symmetric temperature distributions. For example, in models of a circumstellar disk, the disk surface is generally found to be warmer than the disk midplane at the same radial distance from the star.

After determination of the dust temperatures for a multicomponent dust model, a ray-tracing procedure is used to calculate intensity maps for the appearance of the central object and its circumstellar environment at varying inclinations for selected frequencies. The simulated images are then used to compute visibility maps in the $(u, v)$ plane, which finally can be compared to the MIDI measurements. We used 64 wavelength points between $0.1 \mu \mathrm{m}$ and $5 \mathrm{~mm}$ for our modeling of HR 5999. The size of the numerical grid was set to $50 \times 50 \mathrm{AU}$, which resulted in a spatial resolution (i.e. the grid point spacing for the innermost grid) of $0.016 \mathrm{AU}$. As a final check on the numerical accuracy, we used images computed with the ray-tracing procedure at a large number of different inclination angles to determine the total flux (integrated over all wavelengths) as a function of the inclination angle. Integration of these fluxes over all inclination angles then 
gives the total model luminosity, which finally can be compared to the input luminosity. For the geometrical models described below, we find typical deviations of $\$ 1 \%$ in the case of spherical and thick disk models, increasing up to $\sim 8 \%$ for the thinnest disk models (when the vertical thickness of the inner disk gets similar to the numerical grid resolution).

Note that our radiative transfer code not only accounts for extinction by and emission from dust grains, but also treats scattering by dust grains; isotropic scattering is implicitly assumed. Although the scattering efficiency of dust grains is quite low at mid-infrared wavelengths, scattering of optical/UV photons in the region above and below the disk plane can lead to significantly increased heating of the outer disk regions; this can result in more extended thermal emission at mid-infrared wavelengths. If scattering is neglected (as is sometimes done in other radiation transfer simulations), the extent of the emission can be significantly underestimated.

Our radiative transfer modeling assumes the disk is passive (i.e. the temperature at each point is determined by the local radiation field) and does not take into account possible viscous disk heating by accretion. This is appropriate because the stellar parameters and the estimated accretion rate (see Sect. 1) suggest that accretion does not contribute more than $\sim 10 \%$ to the total luminosity.

A self-consistent radiation transfer model should, of course, reproduce not only the observed visibilities, but also the broadband spectral energy distribution (SED). We therefore constructed the SED of HR 5999 from the UV to mm wavelengths from literature data (Hillenbrand et al. 1992; Henning et al. 1994; Acke \& van den Ancker 2004). A complication arises from the presence of the companion Rossiter 3930, which has an angular separation of only 1.4" from HR 5999. The fluxes for the SED points, which were obtained from seeing-limited observations, therefore represent the sum of the fluxes from HR 5999 and Rossiter 3930. The adaptive optics study by Stecklum et al. (1995) showed that the flux of Rossiter 3930 is considerably smaller than that of HR 5999 at optical and near-infrared wavelengths; they determined $\Delta(V, J, H, K) \sim(4.6,3.0,3.1,3.6) \mathrm{mag}$. At longer wavelengths, however, Rossiter 3930 may well contribute a larger fraction to the total flux, and it cannot even be excluded that it may dominate the total flux, as is the case for the several well-known "infrared companions" in binary T Tauri star systems (e.g. Chelli et al. 1988). Although the latter possibility does not appear to be very likely in the case of HR 5999 (since there is no strong increase in the flux contribution from Rossiter 3930 from the optical to the near-infrared range), all SED points longward of $2.2 \mu \mathrm{m}$ can only be considered as upper limits to the flux of HR 5999. In our radiation transfer modeling we therefore cannot require that the model SED matches the observed SED longward of $2.2 \mu \mathrm{m}$, but only that it does not exceed the observed SED. We note that the companion does not affect our MIDI data of HR 5999 since the interferometric field-of-view is smaller than the separation ${ }^{2}$.

As input for the radiation transfer calculations one has to specify the luminosity and effective temperature of the central radiation source (we used $L_{\text {bol }}=87 L_{\odot}$ and $T_{\text {eff }}=7925 \mathrm{~K}$ following van Boekel et al. 2005), the optical properties of the dust, and the density structure of the circumstellar material. The dust composition around HR 5999 was recently investigated by van Boekel et al. (2005), who found that the $8-13 \mu \mathrm{m}$ spectrum is

${ }^{2}$ To be sure, we inspected the acquisition images and found no indication of another source in the MIDI field-of-view. dominated by large silicate (olivine) grains. Guided by their results, we used a dust model here that consists of silicate and carbon grains with sizes of $1 \mu \mathrm{m}$. The sublimation temperature of both grain species is taken to be $1500 \mathrm{~K}$. In the radiation transfer code, the destruction of grains through sublimation is simulated by setting the number density of the grains to zero, if the temperature of that particular grain species, which is calculated simultaneously with the radiation field, exceeds the sublimation temperature.

Model images were computed with the radiation transfer code for those 15 wavelengths between $8 \mu \mathrm{m}$ and $13 \mu \mathrm{m}$ for which the visibilities were determined from the MIDI data. We then calculated visibility maps from the model images and compared them to the measured visibilities for HR 5999. Since the orientation of the disk (i.e. the angle between the disk axis and the north-south direction on the sky, measured counter-clock wise and denoted here as the position angle $\phi$ ) is unknown, an $\chi^{2}$ fitting procedure was used to determine the position angle that results in the best agreement between measured and simulated visibilities. For this we used all 9 visibility curves, each consisting of 15 wavelengths points, i.e. 135 individual data points.

\subsection{Models for the density distribution}

We consider here two different classes of models for the density distribution of the circumstellar material, disk models, and spherical envelope models.

\subsubsection{Extended disk (Model D)}

We started with models of a quasi-Keplerian disk (i.e. the disk structure is determined by the gravitationally dominating central star), with the following density distribution:

$\rho_{\mathrm{D}}=\rho_{0}\left(\frac{r}{r_{0}}\right)^{-p} \exp \left[-\frac{\pi}{4}\left(\frac{z}{h_{z}}\right)^{2}\right]$,

with $h_{z}=\left(\frac{r}{r_{0}}\right)^{q} \times h \cdot r_{0}$.

We used $p=15 / 8$ for the radial density power-law index, and for the radial dependence of the vertical disk scale height $h_{z}$ we assumed $q=9 / 8$, i.e. a slightly flaring disk shape. The parameter $h$ fixes the relative geometrical thickness of the disk.

In our modeling we considered different values for the relative disk thickness $h$ (from very thin disks with $h=0.05$ to very thick disks with $h \sim 0.8$ ), different inclination angles $i$ (from $i=0^{\circ}$ for a face-on view and $i=90^{\circ}$ for an edge-on view), and also tried different values for the radial density power-law index $p$. For each combination of model parameters, the density $\rho_{0}$ is restricted by the requirement that the model SED should agree with the observed SED points for wavelengths $\lambda \leq 2.2 \mu \mathrm{m}$ and must not exceed the observed fluxes for longer wavelengths (where the observed SED points contain an unknown flux contribution from the binary companion).

The resulting brightness distribution in the simulated images is dominated by the central star and the inner disk region of a few AU (see Fig. 6). The peak brightness is found at the inner rim of the dusty disk at the dust sublimation radius, and it drops smoothly outwards. Despite the quite compact appearance of the simulated images, the weak emission from the outer parts of the disk contributes a significant fraction of the total flux at midinfrared wavelengths. This extended emission leads to a large effective size of the emission and yields model visibilities that are 

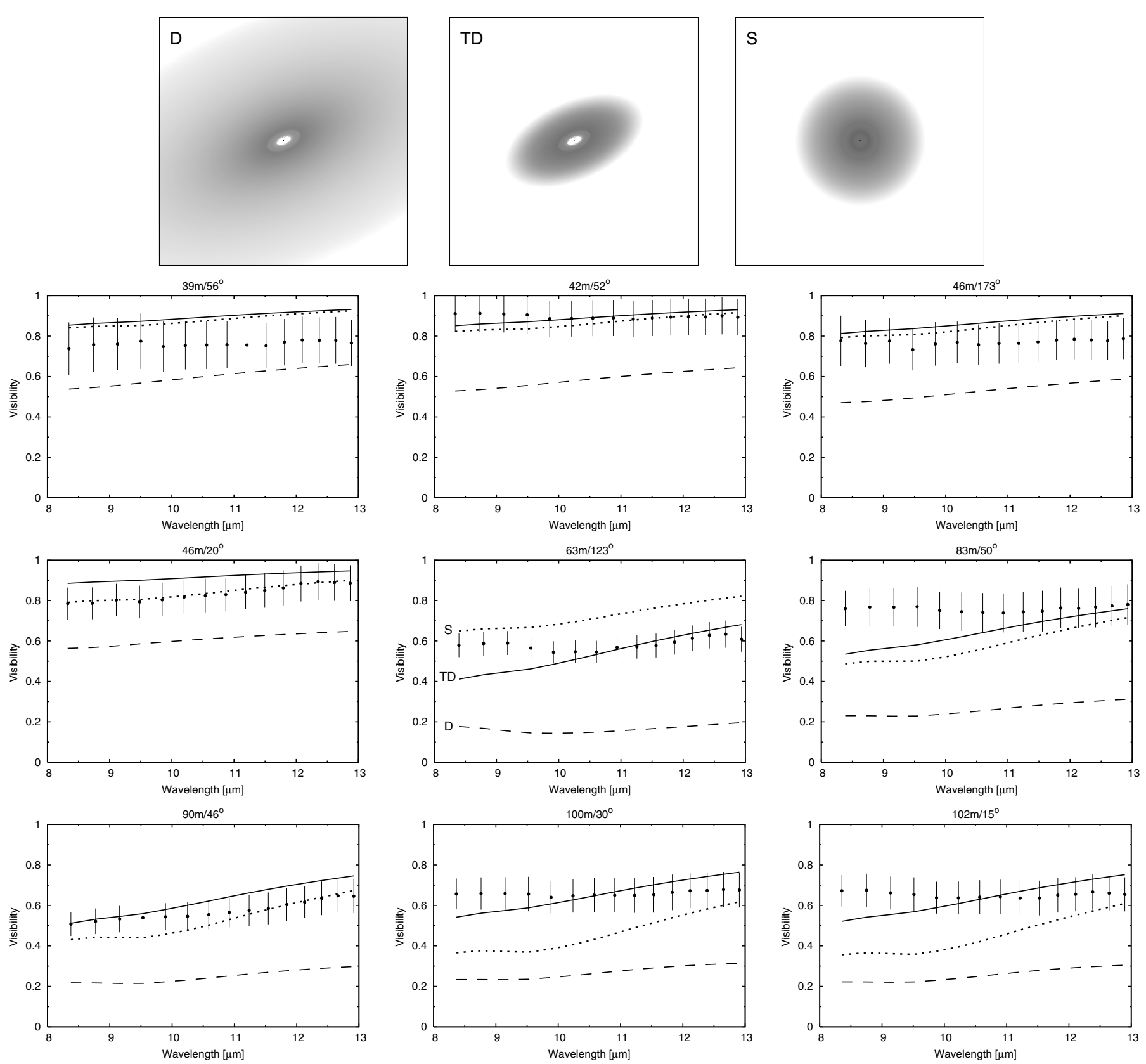

Fig. 6. Radiation transfer modeling of HR 5999. The upper row shows the model images at $10 \mu \mathrm{m}$ for the disk model (D, left), the truncated disk model (TD, middle), and the spherical shell model $(\mathbf{S}$, right). Each image shows a $12.5 \times 12.5$ AU region with a negative logarithmic intensity scale, seen under an inclination angle of $i=59^{\circ}$ and at position angle $\phi=24^{\circ}$. The mosaic of visibility versus wavelength plots compares the observed visibilities of HR 5999 (solid dots with error bars) for each individual MIDI observation to the model visibilities; the truncated disk model (TD) is displayed by the solid line, the disk model without truncation (D) by the dashed line, and the spherical shell model (S) by the dotted line.

considerably lower than some of the observed visibilities. The characteristic size of the model brightness distributions is up to twice as large as the sizes derived from the observed visibilities. Even if we change the power-law exponent for the radial density law (to values as steep as $p=4$ ) or the thickness of the disk, the extent of the emission in the model images is still considerably too large and inconsistent with the observed visibilities.

\subsubsection{Truncated disk (Model TD)}

One possibility for decreasing the amount of extended emission in the outer parts of the images is to limit the circumstellar mass distribution to a certain outer radius. For this, we used the disk model describe above (Eq. (1)) and truncated the density distribution at the outer radius $r_{\mathrm{t}}$. This truncation is performed by multiplying the density distribution with a Fermi-type function:

$\rho_{\mathrm{TD}}=\rho_{\mathrm{D}} \times\left(1+\exp \left[\frac{R-R_{\mathrm{t}}}{\alpha}\right]\right)^{-1}$,

where $\rho_{\mathrm{D}}$ is the density distribution given in Eq. (1), $R=$ $\sqrt{r^{2}+z^{2}}$ is the radial distance from the origin, while $\alpha$ determines the width of the transition zone of the Fermi-type function; we used $\alpha=R_{\mathrm{t}} / 10$.

With these models we were able to find acceptable fits to the observed SED up to $\lambda \sim 10 \mu \mathrm{m}$ (see Fig. 7) and the observed visibilities for truncation radii in the range $\sim 2-3 \mathrm{AU}$, inclination 


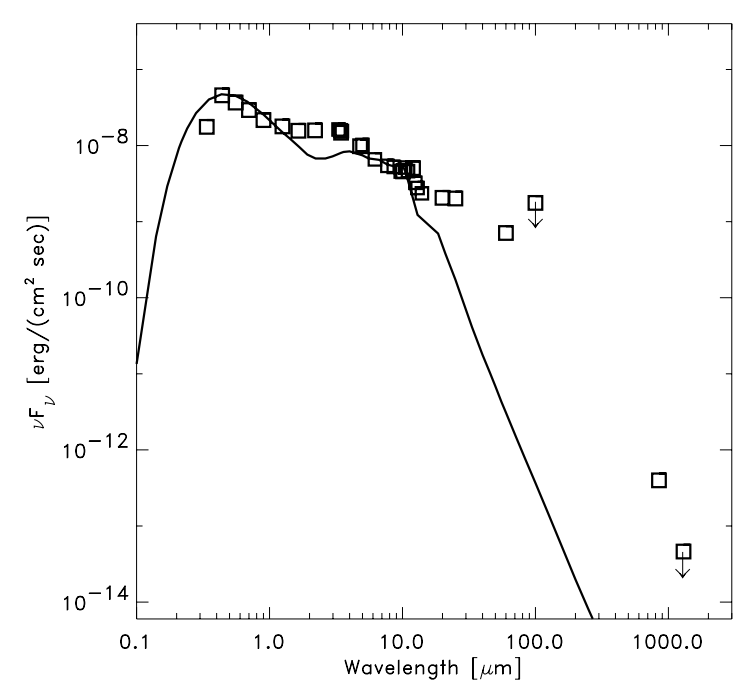

Fig. 7. Observed spectral energy distribution (SED) of HR 599 (boxes; compiled from the references listed in the text) compared the predicted SED for the truncated disk model described in the text. While the model reproduces the SED reasonably well up to $\lambda \sim 10 \mu \mathrm{m}$, it does not account for the bulk of the cool dust responsible for the emission at $\geq 13 \mu \mathrm{m}$. This cool dust may be associated to the companion star Rossiter 3930 or perhaps widely distributed in the HR 5999/R 3930 system. (Note that dust at distances $\geq 100$ AU from HR 5999 would be cool enough to produce excess emission only longward of $10 \mu \mathrm{m}$. The beamsize of the far-infrared SED points corresponds to sizes of several $1000 \mathrm{AU}$, and the data do therefore not allow to constrain the location of the cool dust.)

angles of $i \sim 60^{\circ}$, and position angles of $\phi \sim 25^{\circ}$. The vertical thickness of the disk (governed by the parameter $h$ ) is nearly unconstrained by the data (thin and moderately thick disks seen under intermediate inclination angles "look" very similar).

In Fig. 6 we show a model with parameters $h=0.056$, $R_{\mathrm{t}}=2.6 \mathrm{AU}, i=59^{\circ}$, and $\phi=24^{\circ}$, which yields an acceptable (albeit not perfect) fit to the data with $\chi_{r}^{2}=1.07$. The $V$-band optical depth of this model along the disk plane is $\tau=3.5$. Although models with larger vertical disk scale height give marginally better fits to the visibilities (for example, $\chi_{r}^{2}=0.98$ for a disk with $h=0.4$ ), we prefer this specific thindisk model because the vertical scale height of the dusty disk agrees with the formal condition of vertical hydrostatic equilibrium (see, e.g., Eq. (7), in Chiang \& Goldreich 1997).

For comparison, we note that a model for the same disk parameters but without the truncation factor (model $\mathbf{D}$ in Fig. 6) yields $\chi_{r}^{2}=18.3$.

\subsubsection{Spherical shell (Model S)}

As already shown in Sect. 3.2, the observed visibilities suggest that the brightness distribution is not spherically symmetric but rather somewhat elongated. In order to assess quantitatively how much the truncated disk model provides a better description to the data than spherical shell models, we also considered models with a spherically symmetric power-law density distribution, in which the density distributions were again truncated at radius $R_{\mathrm{t}}$ :

$\rho_{\mathrm{S}}=\rho_{0}\left(\frac{r}{r_{0}}\right)^{-p} \times\left(1+\exp \left[\frac{R-R_{\mathrm{t}}}{\alpha}\right]\right)^{-1}$.

We found that spherical shell models always provide poorer fits to the data than the truncated disk models. A spherical model (model $\mathbf{S}$ in Fig. 6) with the same radial density power-law and truncation radius as in the truncated disk model described above yields $\chi_{r}^{2}=2.91$.

\subsection{Conclusions from the radiative transfer modeling}

We find that dust distributions in which the radial density follows a power law (with exponents in the range $p \sim 1-4$ ) produce brightness distributions that are much too extended to be consistent with our set of MIDI data. Disk models in which the density is truncated at outer radii of $\sim 2-3 \mathrm{AU}$, on the other hand, provide reasonably good fits to the MIDI data.

Given the large number of free parameters in the radiative transfer models and the relatively large visibility errors, which do not allow us to constrain model parameters very well, it was not our aim to scan the full multi-dimensional parameter space in order to find the overall "best fit" model. We instead proceed the other way round and summarize here those model categories that can be ruled out by our data and modeling. The following cases are inconsistent with the MIDI data:

- Spherical shells - The observed visibilities suggest that the brightness distribution is not circular symmetric but rather somewhat elongated. This is confirmed by our radiative transfer modeling, which shows that spherically symmetric shell models cannot produce good fits to the observed data.

- Disks seen at low inclination angles $\left(i<30^{\circ}\right.$, i.e. nearly "face-on") - Such models produce a nearly circular symmetric brightness distribution and thus do not fit the data well.

- Very thick disks $(h>0.7)$ - Such models also produce only very slightly elongated, nearly circular symmetric brightness distributions.

- Geometrically thin disks $(h<0.2)$ seen close to edge-on $\left(i \geq 75^{\circ}\right)$ - Such models predict strongly elongated brightness distributions, which are inconsistent with the moderate amount of asymmetry suggested by the MIDI data.

Finally, we note that there may be other ways to explain the compact brightness distribution. One possibility for decreasing the amount of extended emission in the outer parts of the images may be to introduce a dense puffed-up wall at the inner edge of the dust distribution that casts a shadow on the outer parts of the disk. Such a shadow leads to a much steeper temperature profile in the disk regions behind the rim, and therefore the region containing warm enough dust that radiates significantly in the mid-infrared is smaller than without an inner rim. Models of this kind have, for example, been used by Dullemond et al. (2001) to fit the observed SEDs of HAEBEs. The resulting brightness distribution will depend on the detailed shape of the inner rim, which is not well known (see, e.g., discussion in Isella \& Natta 2005). Since such models introduce several additional free parameters for the radiative transfer modeling, they are beyond the scope of the present study and will be the topic of a forthcoming paper.

\section{Summary and conclusions}

Our MIDI interferometric study has, for the first time, resolved the mid-infrared emission around the Herbig Ae star HR 5999. The most remarkable result is the compactness of the $10 \mu \mathrm{m}$ emission, which has a characteristic radius of only $\sim 2-3 \mathrm{AU}$. The data also suggest that the emitting region is elongated. Our radiative transfer modeling shows that a moderately thick disk with outer edge at $\sim 2.6 \mathrm{AU}$, seen under an intermediate inclination angle, provides a reasonably good description of the data. 
We also find that disk models provide better fits to the data than spherical shell models.

The derived outer disk radius of $\sim 2.6 \mathrm{AU}$ is considerably smaller than canonical assumptions of disk sizes around young stellar objects. This leaves us with the question of why the disk around HR 5999 is so compact. One possible reason may be that the disk was truncated by the gravitational effect of a companion to HR 5999. Dynamical disk clearing is expected to truncate the disks around the stars in a binary system to roughly $1 / 3$ of the orbital separation of the components (see, e.g., the numerical simulations by Pichardo et al. 2005). The presence of the companion Rossiter 3930 is interesting in this context, but the quite large projected separation between HR 5999 and Rossiter 3930 of $\sim 300 \mathrm{AU}$ would require an extremely elliptical orbit in order to explain a disk truncation radius of only $\sim 3$ AU. A more likely possibility would be the presence of another, yet undetected, companion at close separation $(\sim 10$ AU) from HR 5999. The MIDI data provide no hint of the existence of a close companion, but we note that it would essentially be undetectable in the MIDI data if its mid-infrared emission ${ }^{3}$ is less than a few percent of that from the material around HR 5999.

Acknowledgements. We would like to thank the referee for detailed comments that helped to improve this paper.

\section{References}

Acke, B., \& van den Ancker, M. E. 2004, A\&A, 426, 151

Akeson, R. L., Ciardi, D. R., van Belle, G. T., Creech-Eakman, M. J., \& Lada, E. A. 2000, ApJ, 543, 313

Artymowicz, P., \& Lubow, S. H. 1994, ApJ, 421, 651

Blondel, P. F. C., Talavera, A., \& Tjin A Djie, H. R. E. 1993, A\&A, 268, 624

Chiang, E. I., \& Goldreich, P. 1997, ApJ, 490, 368

Chelli, A., Cruz-Gonzalez, I., Zinnecker, H., Carrasco, L., \& Perrier, C. 1988, A\&A, 207, 46

Chesneau, O., Meilland, A., Rivinius, T., et al. 2005a, A\&A, 435, 275

Chesneau, O., Verhoelst, T., Lopez, B., et al. 2005b, A\&A, 435, 563

Dullemond, C. P., Dominik, C., \& Natta, A. 2001, ApJ, 560, 957

Eisner, J. A., Lane, B. F., Akeson, R. L., Hillenbrand, L. A., \& Sargent, A. I. 2003, ApJ, 588, 360

Eisner, J. A., Lane, B. F., Hillenbrand, L. A., Akeson, R. L., \& Sargent, A. I. 2004, ApJ, 613, 1049

Grady, C. A., Woodgate, B. E., Bowers, C. W., et al. 2005, ApJ, 630, 958

Hartmann, L., Kenyon, S. J., \& Calvet, N. 1993, ApJ, 407, 219

Henning, Th., Launhardt, R., Steinacker, J., \& Thamm, E. 1994, A\&A, 291, 546

Herbig, G. H. 1960, ApJS, 4, 337
Herbig, G. H. 1994, in The Nature and Evolutionary Status of Herbig Ae/Be Stars, ed. P. S. Thé, M. R. Pérez, \& E. P. J. van den Heuvel, ASP Conf. Ser. Hillenbrand, L. A., Strom, S. E., Vrba, F. J., \& Keene, J. 1992, ApJ, 397, 613 Hofmann, K.-H., Balega, Y., Preibisch, Th., \& Weigelt, G. 2004, A\&A, 417, 981 Isella, A., \& Natta, A. 2005, A\&A, 438, 899

Jaffe, W. 2004, SPIE Proc., 5491, 715

Leinert Ch., Haas, M., Abraham, P., \& Richichi, A. 2001, A\&A, 375, 927

Leinert, Ch., van Boekel, R., Waters, L. B. F. M., et al. 2004, A\&A, 423, 537

Levermore, C. D., \& Pomraning, G. C. 1981, ApJ, 248, 321

Mannings, V., \& Sargent, A. I. 1997, ApJ, 490, 792

Mannings, V., \& Sargent, A. I. 2000, ApJ, 529, 391

Men'shchikov, A. B., \& Henning, Th. 1994, in Circumstellar Dust Disks and Planet Formation, ed. R. Ferlet, \& A. Vidal-Madjar (Editions Frontières), 381

Millan-Gabet, R., Schloerb, F. P., \& Traub, W. A. 2001, ApJ, 546, 358

Miroshnichenko, A., Ivezix, Z., \& Elitzur, M. 1997, ApJ, 475, L41 (Erratum in ApJ, 479, L153)

Miroshnichenko, A., Ivezix, Z., Vinkovic, D., \& Elitzur, M. 1999, ApJ, 520, L115

Monnier, J. D., \& Millan-Gabet, R. 2002, ApJ, 579, 694

Monnier, J. D., Millan-Gabet, R., Billmeier, R., et al. 2005, ApJ, 624, 832

Natta, A., \& Krügel, E. 1995, A\&A, 302, 849

Natta, A., Prusti, T., Neri, R., et al. 2001, A\&A, 371, 186

Ohnaka, K., Bergeat, J., Driebe, T., et al. 2005, A\&A, 429, 1057

Perez, M. R., Webb, J. R., \& The, P. S. 1992, A\&A, 257, 209

Perez, M. R., Grady, C. A., \& The, P. S. 1993, A\&A, 274, 381

Pichardo, B., Sparke, L. S., \& Aguilar, L. A. 2005, MNRAS, 359, 521

Preibisch, Th., Balega, Y. Y., Schertl, D., Smith, M. D., \& Weigelt, G. 2001, A\&A, 378, 539

Preibisch, Th., Balega, Y., Schertl, D., \& Weigelt, G. 2002, A\&A, 392, 945

Preibisch, Th., Balega, Y., Schertl, D., \& Weigelt, G. 2003, A\&A, 412, 735

Przygodda, F., Chesneau, O., Graser, U., et al. 2003, Ap\&SS, 286, 85

Royer, F., Grenier, S., Baylac, M.-O., Gomez, A. E., \& Zorec, J. 2002, A\&A, 393, 897

Schertl, D., Balega, Y., Hannemann, T., et al. 2000, A\&A, 361, L29

Siebenmorgen, R., Prusti, T., Natta, A., \& Müller, T. G. 2000, A\&A 361, 258

Sonnhalter, C., Preibisch, Th., \& Yorke, H. W. 1995, A\&A, 299, 545

Stecklum, B., Eckart, A., Henning, T., \& Loewe, M. 1995, A\&A, 296, 463

Thé, P. S., de Winter, D., \& Pérez, M. R. 1994, A\&AS, 104, 315

Tuthill, P. G., Monnier, J. D., \& Danchi, W. C. 2001, Nature, 409, 1012

Tuthill, P. G., Monnier, J. D., Danchi, W. C., Hale, D. D. S., \& Townes, C. H. 2002, ApJ, 577, 826

van Boekel, R., Waters, L. B. F. M., Dominik, C., et al. 2003, A\&A, 400, L21

van Boekel, R., Min, M., Leinert, Ch., et al. 2004, Nature, 432, 479

van Boekel, R., Min, M., Waters, L. B. F. M., et al. 2005, A\&A, 437, 189

Vinkovic, D., Ivezic, Z., Jurkic, T., \& Elitzur, M. 2006, ApJ, 636, 348

Weigelt, G., Preibisch, Th., Schertl, D., Balega, Y. Y., \& Smith, M. D. 2002a, A\&A, 381, 905

Weigelt, G., Balega, Y., Hofmann, K.-H., \& Preibisch, Th. 2002b, A\&A, 392, 937

Weigelt, G., Beuther, H., Hofmann, K.-H., et al. 2006, A\&A, 447, 655

Wilkin, F. P., \& Akeson, R. L. 2003, Ap\&SS, 286, 145

\footnotetext{
3 We performed a number of simulations to get a realistic estimate of the MIDI detectability of a possible companion in the following way: To our "best fit" model images (for the truncated disk model) we artificially added a "companion" at a separation of 50 mas from the central star. We considered a range from 1:100 to $1: 1$ for the flux ratio between the emission from the companion and the photospheric emission from the primary star. We then computed the wavelength-dependent visibilities for the $(u, v)$ points of our MIDI observations from these images, and finally determined the difference to the visibilities without the simulated companion. As a general result, we found that the effect of the simulated companion on the visibilities is very small. Even in the case of a very bright companion (flux ratio 1:1), the maximal change in the visibilities was less than $3 \%$, i.e. well below the uncertainty of the MIDI visibilities. We note that, for example, a $1 M_{\odot}$ companion with an age of about $1 \mathrm{Myr}$ would yield a photospheric flux ratio of 1:9. Thus, even if the companion had mid-infrared excess due to its own disk at a level of 10 times the photospheric flux, it would be undetectable in the MIDI data.
} 